\title{
Estimates for the Minimal Width of Polytopes Inscribed in Convex Bodies*
}

\author{
Peter Gritzmann' and Marek Lassak ${ }^{2}$ \\ ${ }^{1}$ Universität Siegen, Fachbereich Mathematik, Hölderlinstrasse 3, \\ D-5900 Siegen, Federal Republic of Germany \\ ${ }^{2}$ Instytut Matematyki i Fizyki ATR, ul. Kaliskiego 7, 85-790 Bydgoszcz, Poland \\ Dedicated to Professor L. Danzer on the occasion of his sixtieth birthday
}

\begin{abstract}
The paper deals with the problem of approximating point sets by $n$-point subsets with respect to the minimal width $w$. Let, in particular, $\mathscr{K}^{d}$ denote the family of all convex bodies in Euclidean $d$-space, let $\mathscr{A} \subset \mathscr{K}^{d}$ and let $n$ be an integer greater than $d$. Then we ask for the greatest number $\mu=\Lambda_{n}(\mathscr{A})$ such that every $A \in \mathscr{A}$ contains a polytope with $n$ vertices which has minimal width at least $\mu w(A)$. We give bounds for $\Lambda_{n}\left(\mathscr{K}^{d}\right)$, for $\Lambda_{n}\left(\mathcal{M}^{d}\right)$, and for $\Lambda_{n}\left(\mathscr{W}^{d}\right)$, where $\mathcal{M}^{d}, \mathscr{W}^{d}$ denote the families of centrally symmetric convex bodies and of bodies of constant width, respectively.
\end{abstract}

\section{Introduction and Results}

Let $\mathbf{E}^{d}$ denote the $d$-dimensional Euclidean space. The scalar product and the Euclidean norm will be denoted by $\langle\cdot\rangle,\|\cdot\|$, respectively, and $\mathbf{B}^{d}, \mathbf{S}^{d-1}$ will indicate the unit ball and the unit sphere in $\mathbf{E}^{d}$. Let $\mathscr{K}^{d}$ denote the family of all convex bodies of $\mathbf{E}^{d}$, this is the family of convex compact sets which have interior points and let $\mathcal{M}^{d}$ denote the family of centrally symmetric convex bodies of $\mathbf{E}^{d}$. The symbols $\mathbb{N}, \mathbb{R}$ denote the sets of positive integers and real numbers, respectively. By $\lfloor\tau\rfloor$ we denote the integer part of a real number. $\tau$.

Now, let $A \subset \mathbf{E}^{d}$. The convex hull of $A$ will be denoted by conv $(A)$. The support functional $s_{A}$ of $A$ is the extended real-valued functional of $\mathbf{E}^{d}$ defined by

$$
s_{A}(z)=\sup \{\langle x, z\rangle \mid x \in A\}
$$

* Research for this paper was conducted in the academic year 1986/87 while both authors were visiting the University of Washington, Seattle. P. Gritzmann was supported by the Alexander von Humboldt Foundation. 
Geometrically, for $C=\operatorname{conv}(A) \in \mathscr{K}^{d}$ and $z \neq 0$, the equation $\langle x, z\rangle=s_{A}(z)$ gives the oriented supporting hyperplane of $C$ normal to $z$ such that the associated positive halfspace does not intersect the interior of $C$. Accordingly, $b_{A}: \mathbf{S}^{d-1} \rightarrow \mathbb{R} \cup$ $\{\infty\}$ defined by

$$
b_{A}(z)=s_{A}(z)+s_{A}(-z)
$$

for $A \neq \varnothing$ and $b_{\wp}(z)=0$ is called the width of $A$. If $K \in \mathscr{K}^{d}$ and $b_{K}$ is constant, $K$ is called a convex body of constant width. The family of convex bodies of $E^{d}$ of constant width is denoted by $\mathscr{W}^{d}$ (a survey of results on $\mathscr{W}^{d}$ is in [4]). The functionals

$$
w(A)=\min \left\{b_{A}(z) \mid z \in \mathbf{S}^{d-1}\right\}, \quad \operatorname{diam}(A)=\max \left\{b_{A}(z) \mid z \in \mathbf{S}^{d-1}\right\}
$$

are called minimal width of $A$ and diameter of $A$, respectively. Since it is natural and convenient we restrict our considerations to the family

$$
\mathscr{S}^{d}=\left\{S \mid S \subset \mathbf{E}^{d} \wedge 0<w(S)<\infty\right\} .
$$

The problem of giving bounds for the minimal width of convex bodies in terms of other functionals which are naturally associated with convex bodies has been investigated for a long time (see, e.g., [2]). There are also computational aspects of questions like this in computer science (see, e.g., [11]).

In the present paper we deal with the following problem.

Problem. For a given family $\mathscr{A} \subset \mathscr{S}^{d}$ and a positive integer $n$ determine the greatest number $\mu=\Lambda_{n}(\mathscr{A})$ such that every $A \in \mathscr{A}$ contains an $n$-point subset $P$ with $w(P) \geq \mu w(A)$.

Let us point out that in the case of $\mathscr{A}=\mathscr{K}^{d}$ we are asking for the quality of approximation of convex bodies by polytopes with $n$ vertices with respect to the minimal width. Even more intuitive, $\Lambda_{n}\left(\mathscr{K}^{d}\right)$ is the maximal number such that every convex body of minimal width 1 contains a polytope with $n$ vertices and minimal width at least $\Lambda_{n}\left(\mathscr{K}^{d}\right)$.

We set, for $S \in \mathscr{S}^{d}$ and $n \in \mathbb{N}$,

$$
\lambda_{n}(S)=\sup \left\{\frac{w(P)}{w(S)}|P \subset S \wedge| P \mid=n\right\} .
$$

Furthermore, for nonempty subfamilies $\mathscr{A}$ of $\mathscr{S}^{d}$ we define

$$
\Lambda_{n}(\mathscr{A})=\inf \left\{\lambda_{n}(A) \mid A \in \mathscr{A}\right\}
$$

We have the following monotonicity relations for $\mathscr{A}, \mathscr{B} \subset \mathscr{G}^{d}$ and $n, m \in \mathbb{N}$ :

$$
\begin{aligned}
& n \leq m \Rightarrow \Lambda_{n}(\mathscr{A}) \leq \Lambda_{m}(\mathscr{A}), \\
& \mathscr{A} \subset \mathscr{B} \Rightarrow \Lambda_{n}(\mathscr{A}) \geq \Lambda_{n}(\mathscr{B}) .
\end{aligned}
$$


In connection with the last implication, observe that if $\mathscr{A} \subset \mathscr{B} \subset \mathscr{S}^{d}$ and if for every $\varepsilon>0$ and every $B \in \mathscr{B}$ there exists a subset $A_{\varepsilon}$ of $B$ in $\mathscr{A}$ such that $w\left(A_{\varepsilon}\right) / w(B) \geq 1-\varepsilon$, then $\Lambda_{n}(\mathscr{A})=\Lambda_{n}(\mathscr{B})$. As we shall see in the next section (see Lemma 2) this implies that for the families $\overline{\mathscr{K}}^{d}$ of all closed convex sets from $\mathscr{S}^{d}$, and $\bar{M}^{d}$ of all centrally symmetric convex closed sets from $\mathscr{T}^{d}$, we have

$$
\Lambda_{n}\left(\overline{\mathscr{K}}^{d}\right)=\Lambda_{n}\left(\mathscr{K}^{d}\right) \text { and } \Lambda_{n}\left(\overline{\mathcal{M}}^{d}\right)=\Lambda_{n}\left(\mathcal{M}^{d}\right)
$$

So we are dealing mainly with the functionals

$$
\Lambda_{n}\left(\mathscr{K}^{d}\right), \quad \Lambda_{n}\left(\mathscr{M}^{d}\right), \quad \text { and } \quad \Lambda_{n}\left(\mathscr{W}^{d}\right)
$$

Clearly, for $d=1$ and $n \geq 2$ all the functionals are 1 and for $n \leq d$ all are 0 . So, in the following we always assume

$$
d \geq 2 \text { and } n \geq d+1 .
$$

Our main results are stated in the following theorem.

Theorem. Here are some properties of $\Lambda_{n}(\mathscr{A})$ :

(1) $\Lambda_{n}\left(\mathscr{P}^{d}\right) \geq \begin{cases}1 /(d+1) & \text { for } d \text { odd }, \\ 1 / d & \text { for d even. }\end{cases}$

(2) $\Lambda_{n}\left(\mathscr{K}^{d}\right) \geq 1 / d$.

(3) $\Lambda_{n}\left(\mathscr{M}^{d}\right)$ is attained for balls and, in particular.

$$
\Lambda_{d+1}\left(M^{d}\right)= \begin{cases}1 / \sqrt{d} & \text { for } d \text { odd } \\ (d+1) /(d \sqrt{d+2}) & \text { for } d \text { even } .\end{cases}
$$

(4) $\Lambda_{3}\left(\mathscr{W}^{2}\right)=0.739 \ldots$ is attained for the Reuleaux pentagon and $\Lambda_{3}\left(\mathscr{K}^{2}\right)>$ 0.583 .

(5) Let $\Delta(k)$ denote the Hausdorff distance between $\mathbf{B}^{d}$ and a best approximating (with respect to the Hausdorff distance) inscribed polytope with $k$ vertices. Then for $n \geq 2(d+1)$ we have

$$
\Lambda_{n}\left(\mathscr{K}^{d}\right) \geq 1-\Delta\left(\left\lfloor\frac{n}{2}\right\rfloor\right),
$$

and there exists a constant $\kappa$ which only depends on $d$ but not on $n$ such that

$$
\Lambda_{n}\left(\mathscr{K}^{d}\right) \geq 1-\kappa n^{-2 /(d-1)} .
$$

Let us close this section with a few comments about our theorem. For $d=2$ and $n=3$ in (1) we have $\Lambda_{3}\left(\mathscr{S}^{2}\right) \geq \frac{1}{2}$. This is a generalization of a property presented in [13]. It was conjectured in [14] that $\Lambda_{3}\left(\mathscr{K}^{2}\right)=0.75$ meaning that the disk is extremal for $\Lambda_{3}\left(\mathscr{K}^{2}\right)$. However, as a consequence of (4) it turns out that this conjecture is false. But the Reuleaux pentagon is not extremal, either. Rather, it seems that $\Lambda_{3}\left(\mathscr{K}^{2}\right)$ might be attained for the regular pentagon. 
Conjecture. $\Lambda_{3}\left(\mathscr{K}^{2}\right)$ is attained only for the regular pentagon, thus

$$
\Lambda_{3}\left(\mathscr{K}^{2}\right)=6 /\left(3+\sqrt{3} \tan 72^{\circ}\right)=0.720 \ldots
$$

A proof of this conjecture would also show $\Lambda_{3}\left(\mathscr{K}^{2}\right)>\Lambda_{3}\left(\mathscr{S}^{2}\right)$.

Let us point out that the second inequality in (5) results from the first by applying asymptotic estimates for the approximation of convex bodies by inscribed polytopes. (However, in terms of the Hausdorff distance the applied estimate is asymptotically best possible (see [3] and [12]) even for $\mathbf{B}^{d}$.) Explicit bounds for $d=2$ are given in [10]. For a survey on approximations of convex bodies see [9].

\section{Some Preliminary Results}

In the sequel we give some auxiliary results, first two lemmas concerning the width of more general sets and then two lemmas concerning the case of simplices. In particular, we begin by completing the justification of restricting our considerations to the bounded case.

Lemma 1. Let $G_{1}, G_{2}, \ldots$, be an increasing sequence of subsets of $E^{d}$ and let $G=\bigcup_{i=1}^{\infty} G_{i}$. Then

$$
w(G)=\sup \left\{w\left(G_{i}\right) \mid i=1,2, \ldots\right\}
$$

Proof. Denote the above supremum by $\omega$. Clearly, $\omega \leq w(G)$. Suppose $\omega$ were smaller than $w(G)$. For each $i \in \mathbb{N}$ there is a $z_{i} \in S^{d-1}$ such that $w\left(G_{i}\right)=b_{G_{1}}\left(z_{i}\right)$. Assume that the sequence $\left(z_{i}\right)_{i \in N}$ converges to some $z \in S^{d-1}$ (if not, we can select a convergent subsequence). By our supposition there is a two-point set $D \subset G$ for which $b_{D}(z)>\omega$. There exists a number $j$ such that $D \subset G_{i}$ for each $i \geq j$. Since $\left(z_{i}\right)_{i \in N}$ converges to $z$, we have $b_{D}\left(z_{k}\right)>\omega$ for some $k \geq j$ and thus $b_{G_{k}}\left(z_{k}\right)>$ $\omega$. But this contradicts $b_{G_{k}}\left(z_{k}\right)=w\left(G_{k}\right) \leq \omega$.

Lemma 2. For every $A \in \mathscr{S}^{d}$ and every $\varepsilon>0$ there is a bounded $A_{\varepsilon} \subset A$ such that

$$
w\left(A_{\varepsilon}\right) \geq(1-\varepsilon) w(A) \text {. }
$$

Proof. Let $G_{i}$ be the intersection of $A$ with the ball of center 0 and radius $i$ for $i=1,2, \ldots$ Of course, $A=\bigcup_{i=1}^{\infty} G_{i}$. By Lemma 1, we can take as $A_{\varepsilon}$ a set $G_{i}$ with $i$ sufficiently large.

Let, for $\alpha \in \mathbb{R}$ and $z \in \mathbf{S}^{d-1}$,

$$
H(z ; \alpha)=\left\{x \in \mathbf{E}^{d} \mid\langle x, z\rangle=\alpha\right\}
$$

and

$$
H^{+}(z ; \alpha)=\left\{x \in \mathbf{E}^{d} \mid\langle x, z\rangle \geq \alpha\right\}, \quad H^{-}(z ; \alpha)=\left\{x \in \mathbf{E}^{d} \mid\langle x, z\rangle \leq \alpha\right\} .
$$

$H(z ; \alpha)$ is the hyperplane orthogonal to $z$ with distance $\alpha$ from the origin, while 
$H^{+}(z ; \alpha), H^{-}(z ; \alpha)$ are the associated halfspaces. Furthermore, let, for $\alpha_{1}, \alpha_{2} \in \mathbb{R}$ with $\alpha_{1} \leq \alpha_{2}$,

$$
S\left(z ; \alpha_{1}, \alpha_{2}\right)=H^{+}\left(z ; \alpha_{1}\right) \cap H^{-}\left(z ; \alpha_{2}\right) .
$$

So, $S\left(z ; \alpha_{1}, \alpha_{2}\right)$ denotes a strip of minimal width $\alpha_{2}-\alpha_{1}$ orthogonal to $z$. Let $A \in \mathscr{S}^{d}$. Then a strip $S=S\left(z ; \alpha_{1}, \alpha_{2}\right)$ is called minimal (with respect to $A$ ) if $A \subset S$ and $w(A)=w(S)$.

Let $T \subset E^{d}$ be a simplex. By the height of $T$ from a vertex $v \in T$ we mean the distance from $v$ to the hyperplane carrying the remaining vertices of $T$. Denote by $h(T)$ the minimal height of $T$.

Lemma 3. For every d-simplex $T \subset E^{d}$ we have

$$
\frac{w(T)}{h(T)} \geq \begin{cases}2 /(d+1) & \text { for } d \text { odd } \\ 2 / d & \text { for d even }\end{cases}
$$

Proof. Enclose $T$ in a strip $S\left(z ; \alpha_{1}, \alpha_{2}\right)$, where $\alpha_{2}-\alpha_{1}=w(T)$. Denote the set of vertices of $T$ by $V$. We may assume without loss of generality that

$$
k=\left|V \cap H\left(z ; \alpha_{1}\right)\right| \leq \begin{cases}(d+1) / 2 & \text { for } d \text { odd } \\ d / 2 & \text { for } d \text { even. }\end{cases}
$$

Let $v_{1}, \ldots, v_{k}=V \cap H\left(z ; \alpha_{1}\right)$. Since $S\left(z ; \alpha_{1}, \alpha_{2}\right)$ is a minimal strip for $T$, there are points $x_{1} \in T \cap H\left(z ; \alpha_{1}\right)$ and $x_{2} \in T \cap H\left(z ; \alpha_{2}\right)$ such that $\left\|x_{2}-x_{1}\right\|=w(T)$. Let $\mu_{1}, \ldots, \mu_{k} \in[0,1]$ with $\sum_{i=1}^{k} \mu_{i}=1$ such that $x_{1}=\sum_{i=1}^{k} \mu_{i} v_{i}$. Assume that $\mu_{1} \geq 1 / k$ and denote by $H$ the hyperplane containing $V \backslash\left\{v_{1}\right\}$. Since $x_{2} \in H$, the distance $\delta\left(x_{1}, H\right)$ of $x_{1}$ and $H$ does not exceed $w(T)$. If $\mu_{1}=1$, we have $h(T)=w(T)$. So consider the case $\mu_{1}<1$. Set

$$
y=\frac{1}{1-\mu_{1}} x_{1}-\frac{\mu_{1}}{1-\mu_{1}} v_{1} .
$$

Obviously, we have $\left\|v_{1}-y\right\| /\left\|x_{1}-y\right\| \leq 1 / \mu_{1} \leq k$. Since $y \in T \cap H$ it follows that $\delta\left(v_{1}, H\right) \leq k w(T)$. Thus $w(T) / h(T) \geq 1 / k$ which proves the assertion.

Let $T$ denote a $d$-simplex in $\mathbf{E}^{d}$ and let $V=V(T)=\left\{v_{0}, \ldots, v_{d}\right\}$ be the set of its vertices. For each $p \in\{1, \ldots, d\}$ set $q=d+1-p$ and define

$$
\mathscr{V}_{p}=\{(P, Q)|P, Q \subset V \wedge P \cap Q=\varnothing \wedge| P|=p \wedge| Q \mid=q\}
$$

Now, let

$$
\zeta_{p}(T)=\sum_{(P, Q) \in \mathscr{V}_{n}}\|\gamma(P)-\gamma(Q)\|^{2}
$$

where

$$
\gamma(P)=\frac{1}{p} \sum_{v, \in P} v_{i}
$$

denotes the center of the face conv $(P)$ (and respectively for $Q$ ). 
Lemma 4. ${ }^{1} \quad$ Let $T$ be an arbitrary d-simplex inscribed in $\mathrm{B}^{d}$ and let $T_{0}$ denote the regular simplex inscribed in $\mathbf{B}^{d}$. Then $w(T) \leq w\left(T_{0}\right)$.

Proof. Let, for $i=0, \ldots, d$ and $j=1, \ldots, d$, the $j$ th coordinate of $v_{i}$ be denoted by $\nu_{i j}$. Since for $(P, Q) \in \mathscr{V}_{p}$ we have

$$
\|\gamma(P)-\gamma(Q)\|^{2}=\sum_{j=1}^{d}\left(\frac{1}{p} \sum_{\nu_{i} \in P} \nu_{i j}-\frac{1}{q} \sum_{\nu_{i} \in Q} \nu_{i j}\right)^{2}
$$

we get

$$
\begin{aligned}
\zeta_{p}(T)= & \frac{1}{p^{2} q^{2}}\left[\left(p^{2}\left(\begin{array}{l}
d \\
p
\end{array}\right)+q^{2}\left(\begin{array}{l}
d \\
q
\end{array}\right)\right) \sum_{i=0}^{d} \sum_{j=1}^{d} \nu_{i j}^{2}\right. \\
& \left.+2 \tau\left(p^{2}\left(\begin{array}{c}
d-1 \\
p
\end{array}\right)+q^{2}\left(\begin{array}{c}
d-1 \\
q
\end{array}\right)-2 p q\left(\begin{array}{c}
d-1 \\
p-1
\end{array}\right)\right)\right]
\end{aligned}
$$

with $\tau=\sum_{j=1}^{d} \sigma_{2}\left(\nu_{0 j}, \ldots, \nu_{d j}\right)$, where $\sigma_{2}(\cdot)$ denotes the second elementary symmetric function. After some simplification (using $\left\|v_{i}\right\|=1$ ) we obtain

$$
\zeta_{p}(T)=\frac{1}{p^{2} q^{2}}\left[p(d+1)^{2}\left(\begin{array}{l}
d \\
p
\end{array}\right)-2 \tau(d+1)\left(\begin{array}{l}
d-1 \\
p-1
\end{array}\right)\right]
$$

Since $\left\|v_{i}\right\|=1$, we get $\tau \geq-(d+1) / 2$. Moreover, $\|\gamma(P)-\gamma(Q)\|^{2}=(d+1)^{2} /(p q d)$ for $(P, Q) \in \mathscr{V}_{p}\left(T_{0}\right)$. Hence,

$$
\zeta_{p}(T) \leq \frac{(d+1)^{2}}{p^{2} q^{2}}\left[p\left(\begin{array}{l}
d \\
p
\end{array}\right)+\left(\begin{array}{c}
d-1 \\
p-1
\end{array}\right)\right]=\frac{(d+1)^{2}}{p^{2} q^{2}}\left(\begin{array}{c}
d+1 \\
p
\end{array}\right)=\zeta_{p}\left(T_{0}\right)
$$

Thus

$$
\sum_{(P, Q) \in \mathcal{V}_{\lfloor(d+1) / 2\rfloor}} w^{2}(T) \leq \zeta_{\lfloor(d+1) / 2\rfloor}(T) \leq \zeta_{\lfloor(d+1) / 2\rfloor}\left(T_{0}\right)=\sum_{(P, Q) \in V_{\lfloor(d+1) / 2\rfloor}} w^{2}\left(T_{0}\right),
$$

which implies $w(T) \leq w\left(T_{0}\right)$.

\section{Proof of the Theorem}

(1) Let $A \in \mathscr{S}^{d}$. For convenience put $\rho=1 /(d+1)$ for $d$ odd and $\rho=1 / d$ for $d$ even.

First consider the case when $\boldsymbol{A}$ is compact. Let $T$ denote a simplex of greatest volume among all simplices with vertices in $A$. Denote by $v$ a vertex of $T$ such

\footnotetext{
'As was pointed out by one of the referees this result has also been obtained by [1].
} 
that the height of $T$ from $v$ is $h(T)$. Let $H(z ; \alpha)$ be the hyperplane carrying all vertices of $T$ except $v$. The maximality of $T$ implies

$$
A \subset S(z ; \alpha-h(T), \alpha+h(T))
$$

Thus $w(A) \leq 2 h(T)$. So by Lemma 3 we get $w(T) / w(A) \geq w(T) / 2 h(T) \geq \rho$.

Now we are ready to work with an arbitrary $A \in \mathscr{P}^{d}$. Let $\varepsilon>0$. By Lemma 2 there is a bounded $A_{\varepsilon} \subset A$ such that

$$
w\left(A_{\varepsilon}\right) \geq(1-\varepsilon) w(A)
$$

Of course, for the closure $\operatorname{cl}\left(A_{\varepsilon}\right)$ of $A_{\varepsilon}$ we have $w\left(\operatorname{cl}\left(A_{\varepsilon}\right)\right)=w\left(A_{\varepsilon}\right)$. Let $T_{\varepsilon}$ be a $d$-simplex of maximal volume with all vertices in $\operatorname{cl}\left(A_{\varepsilon}\right)$. We can find in $A_{\varepsilon}$ a $(d+1)$-point set $P_{\varepsilon}$ such that $\operatorname{conv}\left(P_{\varepsilon}\right)$ contains a translate of $(1-\varepsilon) T_{\varepsilon}$. Hence

$$
w\left(P_{\varepsilon}\right) \geq(1-\varepsilon) w\left(T_{\varepsilon}\right)
$$

From the above inequalities and the compact case considered before we get

$$
\frac{w\left(P_{\varepsilon}\right)}{w(A)} \geq \frac{(1-\varepsilon) w\left(T_{\varepsilon}\right)}{w(A)} \geq(1-\varepsilon)^{2} \frac{w\left(T_{\varepsilon}\right)}{w\left(\operatorname{cl}\left(A_{\varepsilon}\right)\right)} \geq(1-\varepsilon)^{2} \rho
$$

Since $A \in \mathscr{S}^{d}$ and $\varepsilon>0$ are arbitrary, we obtain $\Lambda_{d+1}\left(\mathscr{S}^{d}\right) \geq \rho$.

So (*) implies $\Lambda_{n}\left(\mathscr{S}^{d}\right) \geq \rho$.

(3) Let $K \in \mathscr{M}^{d}$. We may assume that 0 is the center of $K$. We show that $B=\left(\frac{1}{2} w(K)\right) \cdot \mathbf{B}^{d}$ is contained in $K$. Suppose there is a point $b \in \operatorname{int}(B) \backslash K$. Since $K$ is convex there exists a supporting hyperplane $H\left(z_{0} ; \alpha\right)$ through $b$. By symmetry of $K$ we have $K \subset S\left(z_{0} ;-\alpha, \alpha\right)$. But $w\left(S\left(z_{0} ;-\alpha, \alpha\right)\right)=2 \alpha<w(K)$. This contradiction shows that, indeed, $B \subset K$. Thus we have proved that $\Lambda_{n}\left(\mathcal{M}^{d}\right)$ is attained for balls.

Let $T_{0}$ be a regular simplex inscribed $\mathbf{B}^{d}$. An easy calculation shows that

$$
w\left(T_{0}\right)=\|\gamma(P)-\gamma(Q)\|
$$

for each $(P, Q) \in \mathscr{V}_{\{(d+1) / 2\}}\left(T_{0}\right)$, which, in turn, equals the right-hand side of the second assertion of (3). Thus the assertion follows from Lemma 4.

(2) Observe, that by (1) and (**) we already have

$$
\Lambda_{n}\left(\mathscr{K}^{d}\right) \geq \begin{cases}1 /(d+1) & \text { for } d \text { odd } \\ 1 / d & \text { for } d \text { even }\end{cases}
$$

As pointed out by a referee, the improvement from $1 /(d+1)$ to $1 / d$ in case of 
odd $d$ can be obtained as follows. Every $K \in \mathscr{K}^{d}$, with $k$ odd, contains [7, p. 112] a ball of radius $r \geq w(K) /(2 \sqrt{d})$, i.e., of width at least $w(K) / \sqrt{d}$. By (3) the unit ball contains a simplex of minimal width $2 / \sqrt{d}$. These inequalities combined give the lower bound $1 / d$ also for $d$ odd.

(4) As is shown in [8], every $K \in W^{2}$ of width 1 contains an equilateral triangle $T$ of side $l$, where $l$ is the side of a largest equilateral triangle contained in the Reuleaux pentagon of width 1 . Since $0.85338<l<0.85345$, as evaluated by the authors of [8], we have $0.73904<w(T)<0.73911$, which immediately implies the first assertion of (4).

On the other hand, by [6], every plane convex body of minimal width 1 contains a convex body of constant width $1 /(3-\sqrt{3})$. This implies the rest of (4).

(5) Let $K$ be an arbitrary convex body. Let $k=\lfloor n / 2\rfloor$. Further, let $Q$ be a polytope with $k$ vertices $v_{1}, \ldots, v_{k}$ inscribed in $\frac{1}{2} \mathbf{B}^{d}$ such that the Hausdorff distance between $\frac{1}{2} \mathbf{B}^{d}$ and $Q$ is $\Delta(k) / 2$. This means, in particular,

$$
1-\Delta(k) \leq w(Q)
$$

Now, let $\tilde{A}_{i}=\operatorname{conv}\left\{-v_{i}, v_{i}\right\}$, for $i=1, \ldots, k$, and set $\tilde{Q}=\operatorname{conv}\left(\tilde{A_{1}} \cup \cdots \cup \tilde{A}_{k}\right)$. Obviously,

$$
w(Q) \leq w(\tilde{Q})
$$

By $\left[2\right.$, p. 51] there is a segment $A_{i}$ of length greater than or equal to $w(K)$ in $K$ which is parallel to $\tilde{A}_{i}$ for $i=1, \ldots, k$. Let $P=\operatorname{conv}\left(A_{1} \cup \cdots \cup A_{k}\right)$. Then $P$ is a polytope with at most $n$ vertices. In order to estimate the minimum width of $P$ observe that all widths of a convex body do not change under central symmetrization, so in particular for $P^{*}=\frac{1}{2}(P+(-P))$ we have $w(P)=w\left(P^{*}\right)$. Denote by $c_{i}$ the center of $A_{i}$. Set $A_{i}^{\prime}=-c_{i}+A_{i}$ and $P^{\prime}=\operatorname{conv}\left(A_{1}^{\prime} \cup \cdots \cup A_{k}^{\prime}\right)$. Of course, $P^{\prime} \subset P^{*}$. This

$$
w\left(P^{\prime}\right) \leq w\left(P^{*}\right)
$$

Observe that $w(K) \cdot \tilde{Q} \subset P^{\prime}$, which implies

$$
w(K) w(\tilde{Q}) \leq w\left(P^{\prime}\right)
$$

Combining the obtained inequalities we get

$$
w(K)(1-\Delta(k)) \leq w(K) w(Q) \leq w(K) w(\tilde{Q}) \leq w\left(P^{\prime}\right) \leq w\left(P^{*}\right) \leq w(P)
$$

which proves the inequality $\Lambda_{n}\left(\mathscr{K}^{d}\right) \geq 1-\Delta(\lfloor n / 2\rfloor)$. 
The second assertion in (5) follows from the first one and from the fact that, by [5] and [3], every convex body can be approximated by an inscribed polytope with at most $n$ vertices such that the Hausdorff distance is less than

$$
\kappa^{\prime} n^{-2 /(d-1)}
$$

where $\kappa^{\prime}$ is a constant that depends only on the dimension and on the circumradius of $K$.

\section{References}

1. R. Alexander, The width and diameter of a simplex, Geom. Dedicata 6 (1977), 87-94.

2. T. Bonnesen and W. Fenchel, Theorie der konvexen Körper, Springer-Verlag, Berlin, 1934.

3. E. M. Bronstein and L. D. Ivanov, The approximation of convex sets by polyhedra, Siberian Math. J. 16 (1975), 852-853.

4. G. D. Chakerian and H. Groemer, Convex bodies of constant width, in Convexity and Its Applications, ed. by P. M. Gruber and J. M. Wills, Birkhäuser, Basel, 1983, $49-96$.

5. R. Dudley, Metric entropy of some classes of sets with differentiable boundary, J. Approx. Theory 10 (1974), 227-236; Correction ibid. 26 (1979), 192-193.

6. H. G. Eggleston, Sets of constant width contained in a set of given minimal width, Mathematika 2 (1955), 48-55.

7. H. G. Eggleston, Convexity, Cambridge University Press, Cambridge, 1969.

8. H. G. Eggleston and S. J. Taylor, On the size of equilateral triangles which may be inscribed in curves of constant width, $J$. London Math. Soc. 27 (1952), 438-448.

9. P. Gruber. Approximation of convex bodies, in Convexity and Its Applications, ed. by P. M. Gruber and J. M. Wills, Birkhäuser, Basel, 1983, 131-162.

10. D. E. McClure and R. A. Vitale, Polygonal approximation of plane convex bodies, J. Math. Anal. Appl. 51 (1975), 326-335.

11. F. P. Preparata and M. I. Shamos, Computational Geometry, Springer-Verlag, New York, 1985.

12. R. Schneider and J. A. Wieacker, Approximation of convex bodies by polytopes, Bull. London Math. Soc. 13 (1981), 149-156.

13. G. Tsintsifas, Amer. Math. Monthly 83 (1976), 142.

14. G. Tsintsifas, The minimum strip of a point set, Manuscript.

Received September 9, 1987, and in revised form December 14, 1987. 